\title{
Organização do trabalho da enfermagem e assistência integral em saúde
}

Recebido em: 08/12/2010

Julieta Oro ${ }^{1}$

Aceito em: 21/03/2011

Eliane Matos ${ }^{2}$

Revisão integrativa da literatura, trazendo os debates e influências gerados pelos pressupostos de atenção integral à saúde/integralidade na assistência, sobre a organização do trabalho e modelos de distribuição de cuidado em enfermagem. Considerou-se a produção existente na BVS, no período de 2000 a 2010, totalizando 19 artigos. Enfermeiros têm refletido sobre a integralidade a partir das esferas de atenção, da formação profissional e da organização dos serviços, especialmente na saúde coletiva. Conclui-se sobre a necessidade de estudos relacionando a atual organização do trabalho de enfermagem e os métodos de distribuição de cuidados, especialmente no ambiente hospitalar.

Descritores: Assistência Integral à Saúde, Organização e Administração, Enfermagem.

\section{Nursing work organization and integral health care}

Integrative literature review bringing the debates and influences carried out by integral healthcare/ comprehensive care assumptions, about work organization and health care distribution in nursing. It was considered the BVS existing production, from 2000 to 2010 , totalizing 19 abstracts. Nurses have been reflecting about the integrity from the level government health care system, the professional education and the services organization, especially in collective health. The conclusion is that there is a need for further studies related to nursing work and the health care distribution method, especially in the hospital environment.

Descriptors: Integral Health Care, Organization and Administration, Nursing.

\section{Organización del trabajo de enfermería y asistencia integral en salud}

Revisión integrativa de la literatura trayendo los debates e influencias generadas por los presupuestos de atención integral a la salud/integralidad en la asistencia, sobre la organización del trabajo y modelos de distribución de cuidado en enfermería. Esta revisión ha considerado la producción existente en BVS, en el período desde 2000 a 2010, totalizando 19 artículos. Enfermeros han reflexionado sobre la integralidad a partir de las esferas de atención, formación profesional y organización de los servicios, especialmente en la salud colectiva. Se deduce que hay la necesidad de estudios que hagan relación entre la actual organización del trabajo de enfermería y los métodos de distribución de cuidados, especialmente en el ambiente hospitalario.

Descriptores: Asistencia Integral a la Salud, Organización y Administración, Enfermería.

\section{INTRODUÇÃO}

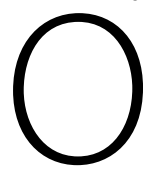

debate acerca do funcionamento do Sistema Único de Saúde (SUS) e da necessidade de melhoria dos serviços prestados à população brasileira coloca em evidência o tema assistência integral/ integralidade da assistência. Integralidade é um conceito com múltiplos entendimentos, dificultando sua apreensão nas realidades concretas do trabalho em saúde; porém, o termo tem sido utilizado para designar "uma das diretrizes básicas do SUS, instituído pela Constituição de 1988", bem como uma "bandeira de luta", um enunciado de certas características do sistema de saúde, de suas instituições e práticas, as quais são

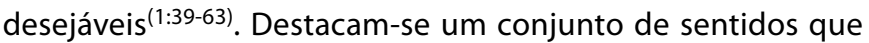
traduzem condições que se aproximam do ideal de assistência integral ou da integralidade da assistência ${ }^{(1,2)}$.
Segundo essa perspectiva, integralidade aproxima-se de uma prática dos profissionais de saúde que se distancia da redução do paciente ao aparelho ou sistema biológico que produziu a doença ou queixa, produzida e reforçada pela prática hegemônica em saúde. Implica em entender o ser humano em sua multidimensionalidade, considerando suas necessidades, desejos, vontades e capacidade de interferir em seu processo de vida e adoecimento ${ }^{(3)}$. Representa a capacidade de repensar o trabalho em rede, a assistência à saúde nas diversas esferas de atenção, integrando os esforços empreendidos em cada nível e ofertando aos usuários assistência contínua. É ainda "relativa às configurações de certas políticas específicas ou especiais" e as formas como essas são conduzidas e diz respeito às políticas governamentais

1 Enfermeira. Mestranda do Mestrado Profissional do Programa de Pós-Graduação em Enfermagem da UFSC. Atua no Hospital Universitário da Universidade Federal de Santa Catarina-UFSC. Email: julieta@hu.ufsc.br.

2 Enfermeira. Doutora em Enfermagem. Professora do Programa de Pós-Graduação de Enfermagem da UFSC. Atua no Hospital Universitário da Universidade Federal de Santa Catarina HU/UFSC. 
desenhadas "para dar resposta a um determinado problema de saúde ou aos problemas de saúde que afligem certo grupo populacional", tanto na perspectiva preventiva quanto assistencial ${ }^{(1: 39-63)}$.

Outros aspectos referem-se à organização dos serviços de saúde para atender às demandas da população por assistência integral, à organização e gestão dos processos de trabalho dos profissionais de saúde, assim como às questões macroestruturais que incluem políticas sociais mais gerais, políticas de saúde específicas e a organização do sistema ${ }^{(1,2)}$.

As políticas de saúde reforçam o protagonismo dos trabalhadores na organização dos serviços e na gestão do trabalho e a necessidade de práticas multiprofissionais que fomentem a inter e a transdisciplinaridade para superar a fragmentação da assistência ${ }^{(2)}$. As mudanças na prática de saúde acontecem tanto nos espaços macrossociais quanto nos microespaços de atuação; porém, o trabalho ainda é predominantemente desarticulado; cada grupo profissional planeja, executa e avalia suas ações isoladamente, sem a necessária articulação interdisciplinar, a qual poderia potencializar a integralidade das ações ${ }^{(3)}$. É necessário repensar o modo como os profissionais de saúde organizam seus processos de trabalho para responder a esses indicativos.

Na enfermagem, tem-se debatido a fragmentação do cuidado a partir do modo de organização do trabalho, que, no Brasil, é agravado pelas condições de trabalho e pela composição da equipe, composta de profissionais com diferentes níveis de formação e com competências diferenciadas para enfermeiros, técnicos e auxiliares de enfermagem. Tradicionalmente, o trabalho é organizado pela distribuição de tarefas parcelares entre os diferentes trabalhadores, considerando a formação e o quadro de pessoal disponível. O enfermeiro realiza os cuidados de maior complexidade e coordena, planeja e avalia os cuidados prestados pela equipe de enfermagem. Esse modo de organização do trabalho compromete a visão do sujeito integral, gerando fragmentação da assistência e afastandose dos indicativos de assistência integral/integralidade ${ }^{(6)}$.

Estudos nacionais que tratam do trabalho da enfermagem apontam dois modos principais de organização e distribuição de cuidados entre os integrantes da equipe de enfermagem: o modelo funcional de distribuição dos cuidados baseia-se na divisão por tarefas. Cada trabalhador executa os cuidados de forma fragmentada, sem articulação com o todo assistencial, distanciando-se do resultado global da assistência e das necessidades da pessoa a ser cuidada ${ }^{(4)}$. "Parte-se das tarefas para padronizar as necessidades dos doentes e não dos doentes para a definição das tarefas. Enfatiza-se a técnica a ser realizada e a necessidade do usuário do serviço se perde em meio ao número de procedimentos a ser executados; o doente se torna tãosomente o 'lugar' em que são realizadas as tarefas"(5: 236); o modelo de distribuição integral dos cuidados representa a prestação de todos os cuidados a um ou mais pacientes por uma única pessoa durante um turno de trabalho. “Esse modelo possibilita uma visão mais global das necessidades do paciente/cliente, tornando o trabalho potencialmente mais criativo", trazendo, ainda que com limites, benefícios para o paciente e para o trabalhador ${ }^{(4: 22)}$.

Os indicativos de integralidade da assistência no âmbito do SUS e a compreensão do papel da enfermagem na construção dessa prática nos levaram a buscar identificar na literatura nacional de que modo a enfermagem tem repensado os modos de organização do trabalho e a distribuição dos cuidados entre os integrantes da equipe de enfermagem.

\section{METODOLOGIA}

Revisão integrativa com abordagem qualitativa que investiga a produção do conhecimento de enfermagem envolvendo os indicativos das políticas públicas de construção da integralidade e assistência integral aos usuários do Sistema Único de Saúde (SUS) e os modelos de distribuição de cuidados entre os trabalhadores de enfermagem.

A revisão integrativa de literatura contribui para compreender melhor um determinado tema e para o desenvolvimento do conhecimento em áreas especializadas. Seguindo normas de clareza e de rigor científico, consideraram-se as seguintes etapas na realização do estudo: identificação do tema e formulação da hipótese; estabelecimento de critérios de inclusão e exclusão; definição das informações a serem extraídas dos estudos selecionados; análise dos estudos; discussão e apresentação dos resultados ${ }^{(5)}$.

A busca de material foi realizada na base de dados da Biblioteca Virtual em Saúde (BVS), que contém publicações das fontes Ciências da Saúde em Geral, utilizando combinações de expressões de modo a atingir os objetivos desejados: cuidado integral, cuidado funcional, enfermagem; integralidade, método de distribuição do cuidado; organização e divisão do trabalho e equipe de assistência ao paciente.

A busca do material foi realizada pelo acesso on-line, em maio e junho de 2010, resultando na captação de 147 artigos. Seguindo os critérios de inclusão e exclusão, 19 foram selecionados para o estudo.

Os critérios de inclusão utilizados foram: artigos completos publicados em português, com resultados disponíveis on-line, período estabelecido entre janeiro de 2000 e janeiro de 2010; foram excluídos os artigos duplicados e os publicados fora do período estipulado.

Para a análise dos artigos, foi elaborado um instrumento contemplando: identificação do artigo original, autores, local e sujeitos da pesquisa, metodologia utilizada, resultados e 
conclusões/recomendações, que orientou a apresentação e discussão dos dados.

\section{RESULTADOS E ANÁLISE DOS DADOS}

Os artigos analisados mostraram que a enfermagem tem debatido o tema integralidade na assistência em saúde, especialmente no campo da saúde coletiva, fazendo algumas relações com a organização do trabalho.

Onze artigos $(57,89 \%)$ referem-se à atuação da equipe de saúde e de enfermagem nas Unidades Básicas de Saúde (UBS), na Estratégia de Saúde da Família (ESF) e equipes de referencia em saúde mental. Seis artigos (31,57\%) tratam da integralidade na assistência hospitalar e três (15,78\%) discutem a formação profissional. Nenhum estudo aborda a organização do trabalho da enfermagem e os métodos de distribuição de cuidados fazendo relação com a perspectiva de assistência integral ou a organização/divisão do trabalho internamente à enfermagem como um dos elementos de uma boa prática, que potencializem a assistência integral e a integralidade.

Um primeiro aspecto abordado na produção de enfermagem diz respeito aos entendimentos dos enfermeiros acerca da integralidade, vista como um modo de cuidar que "valoriza a vida e respeita as diferenças"(7). Enfermeiros defendem o atendimento integral aos usuários do SUS, porém não conseguem conceituálo no contexto profissional nem na prática assistencial ${ }^{(8)}$. A compreensão da integralidade do cuidado na perspectiva de um modelo de atenção à saúde centrado no usuário suscita reflexões sobre as tecnologias e a organização do trabalho, colocando-se como desafio com repercussão na resolutividade, qualidade e humanização dos serviços, e implica em perceber contextualizadamente os usuários em suas múltiplas dimensões ${ }^{(9,10)}$.

Outro aspecto diz respeito às potencialidades da integralidade para a melhoria das ações de saúde e limitações dos profissionais e serviços para sua concretização na realidade.

Destacam-se as dificuldades existentes para a concretização do atendimento integral nos serviços; a inexistência de uma organização voltada para o atendimento integral do indivíduo e da comunidade; o despreparo dos profissionais para tornar a prática e o sistema integralizados; a desorganização do serviço e o aparente conformismo dos profissionais, dificultando ainda mais essa prática ${ }^{(9,10,11)}$.

Estudos envolvendo a percepção dos usuários quanto ao atendimento recebido confirmam a fragilidade da atuação dos profissionais para a efetivação da integralidade, considerando a desarticulação da atenção, a pouca interação e o despreparo dos profissionais para lidar com a dimensão subjetiva da atenção à saúde ${ }^{(7,8,11,12)}$.

Quanto à integração entre as esferas de atenção e a integralidade da assistência, salientam-se as mudanças que vêm ocorrendo nos últimos anos na organização dos serviços de saúde, apontando para uma transição de um cuidado hospitalocêntrico para um cuidado mais integral e humanizado, especialmente em áreas como a saúde mental ${ }^{(13)}$. No ambiente hospitalar, é possível contribuir para a integralidade da atenção quando se percebe o ser humano em sua multidimensionalidade ${ }^{(14)}$

Sobre a formação e capacitação dos profissionais para o exercício da integralidade, o movimento que incita ao trabalho multiprofissional com características interdisciplinares tem perseguido uma aprendizagem voltada à abordagem integral das necessidades de saúde da população, indo além do âmbito individual-biológico(15). Nesse sentido, embora a formação do enfermeiro já sinalize para mudanças, os conteúdos e programas desenvolvidos nos cursos de graduação têm se mostrado insuficientes e/ou desarticulados com as práticas integrais de cuidado ${ }^{(16)}$. As práticas pedagógicas e assistenciais que reiteram o modelo biomédico prevalecem na realidade e o ensino acadêmico não consegue dar conta de uma formação que capacite os profissionais para trabalhar a integralidade na prática e na organização do serviço. Aparentemente, as características do serviço parecem reduzir, com o decorrer do tempo, a iniciativa e a visão ampliadas do ser humano ${ }^{(8,9)}$.

Um desafio para a formação dos enfermeiros é o resgate das práticas cuidadoras do núcleo profissional específico e das intersecções no campo da saúde, valorizando a aprendizagem que estimula a vivência e a reflexão sobre o processo de cuidar ${ }^{(17)}$.

Sobre a formação do técnico de enfermagem, destaca-se o desprestígio da formação/ capacitação desse profissional e salienta-se a importância de sua valorização na equipe de saúde, uma vez que ele realiza a maior parte dos cuidados diretos ao doente institucionalizado. Essa condição compromete pela não-instrumentalização, a prestação de uma assistência mais integradora ${ }^{(18)}$.

Quanto à organização do trabalho em saúde e a construção da integralidade, alguns avanços são apontados para as mudanças na prática, como, por exemplo, a consulta coletiva, realizada na atenção básica, pois contribui para a ruptura do paradigma assistencial-biomédico e com uma assistência que valoriza o usuário(19).

De modo geral, a atenção integral e a integralidade são percebidas como uma utopia, parte do discurso oficial de reorganização da assistência, que ainda não se materializou nas práticas assistenciais, em que não se consegue demonstrar resultados concretos $^{(12)}$. Predomina na prática a desarticulação e fragmentação da assistência, e os enfermeiros entendem que a superação da realidade depende do trabalho em equipe, crítico e reflexivo, com articulação e integração das equipes assistenciais ${ }^{(16-18,20)}$. 


\section{CONSIDERAÇÕES FINAIS}

Evidencia-se, nas últimas décadas, na literatura nacional, volume crescente de produções, que colocam a enfermagem no cenário das discussões quanto às práticas em saúde e à construção da integralidade da assistência.

O centro desse debate situa-se na compreensão sobre os sentidos da integralidade, o (des)preparo dos profissionais, as limitações da formação e da organização dos serviços e do processo de trabalho dos profissionais para sua efetivação na realidade. Apontam-se as principais dificuldades e facilidades enfrentadas no cotidiano para a percepção do usuário em sua multidimensionalidade, com necessidades que exigem respostas complexas a seus problemas de saúde/doença.

É frágil a discussão acerca da organização interna do trabalho da enfermagem com vistas a repensar os modos de organização do trabalho e métodos de distribuição de cuidados entre os trabalhadores de enfermagem enquanto espaço de construção da atenção integral aos usuários dos serviços de saúde, especialmente no meio hospitalar, onde a assistência de enfermagem ocorre de modo mais fragmentado. Novos estudos são necessários visando a repensar a organização do trabalho na categoria e a relação entre modos de distribuição de cuidados e integralidade da assistência.

\section{Referências}

1. Mattos RA. Os sentidos da integralidade: algumas reflexōes acerca dos valores que merecem ser defendidos. In: Pinheiro R, Mattos RA, organizadores. Os sentidos da integralidade na atenção e no cuidado à saúde. Rio de Janeiro: Uerj/IMS/Abrasco; 2001. p. 39-64.

2. Ministério da Saúde (BR). HumanizaSUS: Política Nacional de Humanização: a humanização como eixo norteador das práticas de atenção e gestão em todas as instâncias do SUS. Brasília: Ministério da Saúde; 2004.

3. Matos E, Pires DEP. Práticas de cuidado na perspectiva interdisciplinar: um caminho promissor. Texto Contexto Enferm. 2009;18(2):338-46.

4. Pires DEP. A estrutura objetiva do trabalho em saúde. In: Leopardi

MT, organizador. Organização do trabalho em saúde: organização e subjetividade. Florianópolis: Papa-Livros; 1999. p. 25-48.

5. Costa JS. Métodos de prestação de cuidados [Internet]. [citado em 2010 Jul 10]. Disponível em: http://www.jpv.pt/millenium/Millenium30/19.pdf. 6. Ganong LH. Integrative review of nursing research. Res Nurs Health. 1987;10(1):1-11.

7. Pinho IC, Siqueira JCBA, Pinho LMO. As percepções do enfermeiro acerca da integralidade da assistência [Internet]. [citado em 2010 Jul 10]. Disponível: http://www.fen.ufg.br/revista/revista8_1/original_05.htm. 8. Reis CB, Andrade SMO. Representações sociais das enfermeiras sobre a integralidade na assistência à saúde da mulher na rede básica. Ciênc Saúde Coletiva. 2008;13(1):61-70.

9. Silva KL, Sena RR. Integralidade do cuidado na saúde: indicações a partir da formação do enfermeiro. Rev Esc Enferm USP. 2008;42(1):48-56.

10. Beck CLC, Lisbôa RL, Tavares JP, Silva RM, Prestes FC. Humanização da assistência de enfermagem: percepção de enfermeiros nos serviços de saúde de um município. Rev Gaúcha Enferm. 2009;30(1):54-61.

11. Duarte ED, Sena RR, Xavier CC. Processo de trabalho na Unidade de Terapia Intensiva Neonatal: construção de uma atenção orientada pela integralidade. Rev Esc Enferm USP. 2009;43(3):647-54.

12. Urbano GB. Integralidade na prática assistencial da equipe de enfermagem: a relação da escuta na interação usuário e profissional no município de Paranavaí-PR [dissertação]. Rio de Janeiro: Universidade do Estado do Rio de Janeiro; 2009.

13. Calgaro A, Souza EN. Percepção do enfermeiro acerca da prática assistencial nos serviços públicos extra-hospitalares de saúde mental. Rev Gaúcha Enferm. 2009;30(3):476-83.

14. Morais GSN, Costa SFG, Fontes WD, Carneiro AD. Comunicação como instrumento básico no cuidar humanizado em enfermagem ao paciente hospitalizado. Acta Paul Enferm. 2009;22(3):323-7.

15. Ferreira RC, Vargas CRR, Silva RF. Trabalho em equipe multiprofissional: a perspectiva dos residentes médicos em saúde da família. Ciênc Saúde Coletiva. 2009;4(1):1421-8.

16. Olschowsky A, Duarte MLC. Saberes dos enfermeiros em uma unidade de internação psiquiátrica de um hospital universitário. Rev Latinoam Enferm. 2007;15(4):689-91.

17. Sena RR, Silva KL, Gonçalves AM, Duaret ED, Coelho S. O cuidado no trabalho em saúde: implicações para a formação de enfermeiros. Interface Comum Saúde Educ. 2008;12(24):23-34.

18. Avena DA. O engenho por dentro: cartografia das práticas cotidianas de cuidado em saúde mental dos auxiliares e técnicos de enfermagem na perspectiva da integralidade [dissertação]. Rio de Janeiro: Universidade Federal do Rio de Janeiro; 2009.

19. Penna LHG, Carinhanha Jl, Rodrigues RF. Consulta coletiva de pré-natal: uma nova proposta para uma assistência integral. Rev Latinoam Enferm. 2008;16(1):158-60.

20. Marques D, Silva EM. A enfermagem e o Programa Saúde da Família: uma parceria de sucesso? Rev Bras Enferm. 2004;57(5):545-50. 